\title{
OPEN Comparison of the amniotic fluid and fetal urine peptidome for biomarker discovery in renal developmental disease
}

\author{
Camille Fédou ${ }^{1,2}$, Benjamin Breuil ${ }^{1,2}$, Igor Golovko3 ${ }^{3}$, Stéphane Decramer ${ }^{1,2,4}$, \\ Pedro Magalhães ${ }^{3}$, Françoise Muller ${ }^{5}$, Sophie Dreux ${ }^{5}$, Petra Zürbig ${ }^{3}$, Julie Klein ${ }^{1,2}$, \\ Joost P. Schanstra ${ }^{1,2 \square}$ \& Bénédicte Buffin-Meyer ${ }^{1,2 \square}$
}

Production of amniotic fluid (AF) is view as predominately driven by excretion of fetal urine (FU). However, the origin of AF peptides, often considered as potential biomarkers of developmental diseases, has never been investigated. Here, we evaluated the FU origin of AF peptides and if the AF peptide content can be used as a surrogate of FU. The abundance of endogenous peptides was analyzed by capillary electrophoresis coupled to mass spectrometry in $216 \mathrm{AF}$ and $64 \mathrm{FU}$ samples. A total of 2668 and 3257 peptides was found in AF and FU respectively. The AF peptidome largely overlapped with the FU peptidome, ranging from $54 \%$ in the second pregnancy trimester to $65 \%$ in the third trimester. Examination of a subset of 16 paired AF and FU samples revealed that 67 peptides displayed a significant positively correlated abundance in AF and FU, strongly suggesting that their presence in AF was directly associated to FU excretion. As proof-of-concept we showed that measuring the AF abundance of these 67 peptides of FU origin allowed prediction of postnatal renal survival in fetuses with posterior urethral valves. These results demonstrate that the AF peptidome can be considered as a good surrogate of the FU peptidome.

Exploring the urinary peptidome ("peptidomics") in both adults and children led to the identification of peptidebased signatures allowing diagnosis/prognosis of diseases including kidney ${ }^{1-4}$ or cardiovascular diseases $^{5-7}$, as well as cancers ${ }^{8,9}$. Peptidomics has also been successfully applied in the prenatal context. We identified and validated 12 peptides in fetal urine (FU) that predicted in utero postnatal renal survival in fetuses with developmental renal disease (i.e. posterior urethral valves, PUV ${ }^{10,11}$. However, in contrast to postnatal urine, sampling of FU is invasive since it is collected directly from the fetal bladder. Amniotic fluid (AF) can also be used as a reliable source of biomarkers for fetal diseases ${ }^{12}$. We found a panel of $34 \mathrm{AF}$ peptides that predicted the neurological severity of congenital cytomegalovirus (CMV) infection ${ }^{13}$. More recently, using a prospective multicentre cohort including 178 patients, we identified an AF signature containing 98 peptides that predicted with high accuracy the postnatal renal outcome in fetuses having bilateral kidney malformations (CAKUT for congenital anomalies of the kidney and urinary tract), outperforming predictions based on currently used clinical methods ${ }^{14}$. The presence of such markers in AF is thus particularly interesting since AF sampling is less invasive than FU collection.

$\mathrm{AF}$ is depending on both fetal and maternal tissues. During the first half of the pregnancy, AF is the transudate of plasma from the fetus across the not-yet-keratinized fetal skin or from the mother across the placenta surface. In the second half of the pregnancy, when the fetal skin is fully keratinized, AF production results from fetal urine excretion and fetal pulmonary fluid secretion whereas AF removal is mainly driven by fetal swallowing and transfer to the fetal circulation across the amniotic membrane ${ }^{15,16}$. Comparison of the protein content of $\mathrm{AF}$ and (adult) urine showed that $73 \%$ of proteins present in AF were also detected in urine ${ }^{17}$. However, no data is available regarding the overlap between $\mathrm{FU}$ and $\mathrm{AF}$ peptides with the aim to determine whether AF peptides could be used as a surrogate for FU peptides.

${ }^{1}$ Institut National de La Santé Et de La Recherche Médicale (INSERM), U1048/I2MC-Equipe 12, Institut of Cardiovascular and Metabolic Disease, 1 Avenue Jean Poulhès, BP 84225, 31432 Toulouse Cedex 4, France. ${ }^{2}$ Université Toulouse III Paul-Sabatier, Toulouse, France. ${ }^{3}$ Mosaiques Diagnostics GmbH, Hannover, Germany. ${ }^{4}$ Hôpital Des Enfants, Service de Néphrologie - Médecine Interne - Hypertension Pédiatrique, CHU Toulouse, Toulouse, France. ${ }^{5}$ Unité de Biochimie Fœto-Placentaire, Laboratoire de Biochimie - Hormonologie CHU Robert Debré, AP-HP, Paris, France. ${ }^{\circledR}$ email: joost-peter.schanstra@inserm.fr; benedicte.buffin-meyer@inserm.fr 
Here we investigated the origin of AF peptides by performing a comprehensive analysis of AF and FU peptidome depending on gestational age. A total of $280 \mathrm{AF}$ and FU samples analysed for peptide profiles using capillary electrophoresis coupled to mass spectrometry (CE-MS) were compared. The overlap of peptides present in these two body fluids together with their correlation in abundance were evaluated. Finally, as a proof-of-concept, the potential of quantifying the abundance of AF peptides of FU origin for predicting fetal renal disease progression was assessed.

\section{Results}

Two hundred eighty fetuses with gestational age extending from 11 to 39 weeks of amenorrhea (WA) (Fig. S1A) were included from retrospective CE-MS-based peptidomics studies ${ }^{10,13,14,18}$. For AF analysis, 216 patients were included (Fig. S1B): thirteen fetuses were healthy, 47 were CMV infected and 156 had CAKUT. The major CAKUT aetiology was lower urinary tract obstruction mainly associated with PUV (45/156, Table S1). For FU analysis, 64 patients were included (Fig. S1C): all were CAKUT fetuses with PUV. For a subset of 16 patients, paired FU and AF samples were available.

AF and FU peptide content. A total of 2668 peptides were detected in AF whereas 3257 peptides were found in FU (Fig. 1A). Despite this lower molecular diversity, average abundance of peptides in AF was higher than in FU (Fig. 1A). In both body fluids, $90 \%$ of the detected peptides had a mass comprised between 800 and $5000 \mathrm{Da}$ (Fig. 1B). However, the molecular mass of the peptides was slightly lower in AF (means \pm SEM: $2945+/-38 \mathrm{Da}$ in AF versus $3110+/-36 \mathrm{Da}$ in FU; $\mathrm{p}<0.0001$ ). Sequence information was available for 607 and 620 peptides in AF and FU, respectively. The majority of peptides originated from various collagens $(\sim 75 \%)$. The other most frequently found peptides included fragments of insulin-like growth factor with associated binding protein $(\sim 4 \%)$ or fibrinogen $(\sim 3 \%)$ followed by peptides derived from osteopontin, apolipoprotein, hemoglobin or inter-alpha-trypsin inhibitor heavy chain H4. The most abundant AF peptides (top 2.5\%) included 11 fragments of collagen (together covering $4 \%$ of the total detected peptide signal), 4 fragments of thymosin beta 4 ( $1 \%$ of total signal), 1 cornulin peptide and 1 heparin cofactor 2 fragment. In contrast, the most abundant FU peptides (top 2.5\%) were all derived from collagen and explained $12 \%$ of the total peptide intensity. The number of peptides in AF increased with gestational age until 29-31 WA and then stabilized whereas in FU no increase in the number of peptides was observed, except in late pregnancy (Fig. 1C). In both body fluids, a reduction of peptide abundance was observed with increasing gestational age (Fig. 1D).

AF and FU peptidome overlap. Analysis of the overlap in AF and FU peptidome over the full gestational period led to the identification of 1831 peptides detectable in both AF and FU (Fig. 2A) representing 69\% of the total number and $72 \%$ of total abundance of AF peptides. This suggests that an important fraction of AF peptides might originate from FU. The majority of common sequenced peptides were various collagens (383 peptides) or other proteins including fibrinogen (10 peptides), insulin-like growth factor (8 peptides) and insulin-like growth factor-binding protein (8 peptides) (Fig. 2B). The number of common peptides increased with gestational age, and the part of AF peptidome which overlapped with FU peptidome increased from 54 to $65 \%$ between the second and the third pregnancy trimester (Fig. 2C,D).

Correlation of overlapping peptides in AF and FU. A strong positive correlation between the average abundance of 1831 overlapping peptides in AF and the average abundance in FU was found $(\mathrm{r}=0.58, \mathrm{p}<0.0001)$ (Fig. 3A), thereby indicating that common peptidome was globally correlated in the two fluids. This correlation was mainly driven to overlapping collagen fragments $(\mathrm{r}=0.70, \mathrm{p}<0.0001)$, particularly peptides that issued from collagen alpha-1 (I) chain $(\mathrm{r}=0.79, \mathrm{p}<0.0001)$.

Next, using the subset of 16 paired AF and FU samples, we identified 67 common peptides whose abundance in AF was significantly positively correlated to peptide abundance in FU (Fig. 3B). These AF peptides were thus considered as having predominantly a FU origin. They included different fragments of collagen (15 peptides) and osteopontin (1 peptide) (Table S2). For example, peptides 18625 and 5116 (both derived from Collagen alpha-1(I) chain), as well as peptides 48151 and 28320 displayed high correlation coefficients (Fig. 3C).

AF biomarkers for fetal diseases. As shown here, since FU is the primary source for the AF peptidome, we hypothesized that measuring predominantly FU-derived peptides in AF could be used as a surrogate for FU peptides to prognosticate renal developmental disease, thereby reducing the invasiveness of the procedure to obtain the desired body fluid. As a proof-of-concept study, we tested this hypothesis for the prediction of postnatal renal survival in a small set of 30 fetuses with PUV (Table S3).

Focusing on the $67 \mathrm{AF} / \mathrm{FU}$ common and correlated peptides in a discovery cohort of 14 PUV patients (6 with early ESRD and 8 without ESRD at 2 years postnatally (noESRD)) yielded 7 peptides with significantly different abundance in AF. The 7 peptides were included in a random forest discrimination model (called 'AF7cPUV') which was optimized for the separation of the two patient populations. The classifier was next validated in a test cohort of 16 other PUV fetuses ( 8 ESRD and 8 noESRD patients with matched AF and FU samples). This validation yielded a prediction of postnatal renal survival with $100 \%$ sensitivity $[95 \%$ confidence interval CI: 63-100], 87.5\% specificity [95\%CI: 47-100] and an area under the receiver-operating-characteristic curve (AUC) of 0.97 [95\%CI: 0.89-1] (Fig. 4A,B). The AF7cPUV based prediction was similar to the performance of a previously published classifier including 12 fetal urinary peptides ('(FU)12PUV'; sensitivity 87.5\% [95\%CI: 47-100], specificity 100\% [95\%CI: 63-100], AUC 0.97 [95\%CI: 0.89-1] $)^{10}$ (Fig. 4A,B). In addition, the AF7cPUV and 12PUV scores for these patients were significantly correlated (Fig. 4C). Thus, the current proof-of-concept study supports the use of AF in fetal disease as a surrogate of FU. 
A

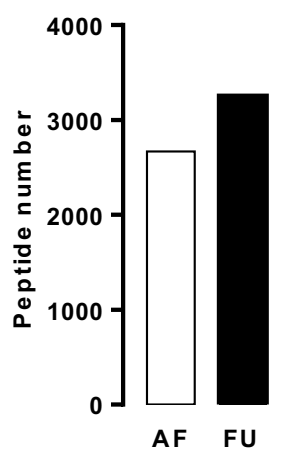

C
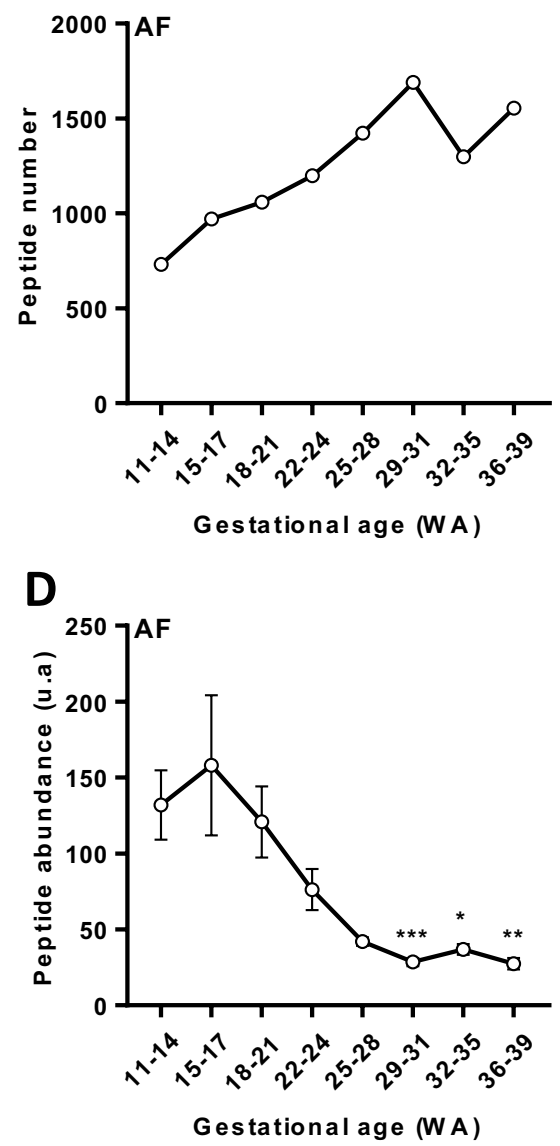

B
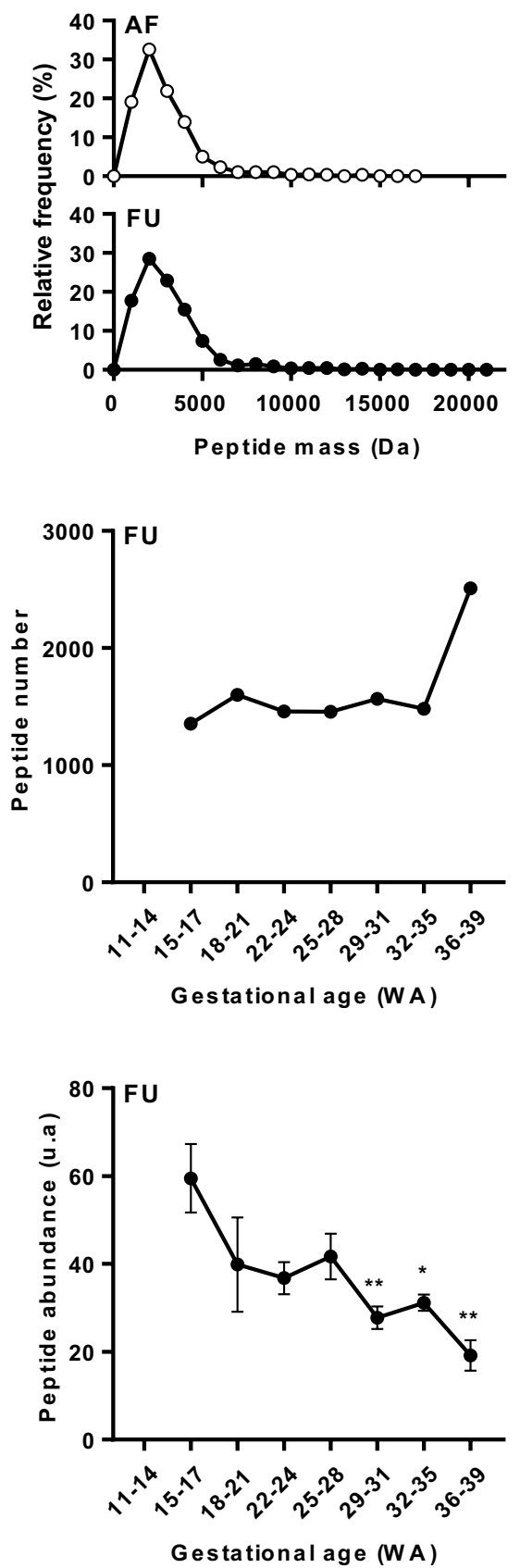

Figure 1. Amniotic fluid and fetal urine peptidome and evolution with gestational age. (A) Number and abundance of peptides in amniotic fluid (AF) and fetal urine (FU) irrespective of gestational age. (B) Weight distribution of peptides present in $\mathrm{AF}$ and FU. The mean and the median were respectively $2945 \mathrm{Da}$ and $2425 \mathrm{Da}$ for AF peptidome and $3110 \mathrm{Da}$ and $2647 \mathrm{Da}$ for FU peptidome. (C) Gestational age evolution of peptide number in AF (left panel) and FU (right panel). (D) Gestational age evolution of peptide abundance in AF (left panel) and FU (right panel). Data are means \pm SEM. ${ }^{*} \mathrm{p}<0.05,{ }^{* *} \mathrm{p}<0.01,{ }^{* *} \mathrm{p}<0.001$ versus $11-14 \mathrm{WA}$ (AF) or 15-17 WA (FU), according to Mann-Whitney test (A) or One-way Anova (D).

\section{Discussion}

The present study compared the AF and FU peptide profiles of 280 fetuses with the aim to determine the origin of the AF peptidome. As main result, we observed that AF peptidome largely overlaps with FU (from $54 \%$ in the second trimester to $65 \%$ in the third pregnancy trimester), thereby suggesting that an important fraction of amniotic peptides originates, at least in part, from FU. A proof-of-concept was provided for the use of the AF peptidome as a source of surrogate biomarkers of FU origin. 
A

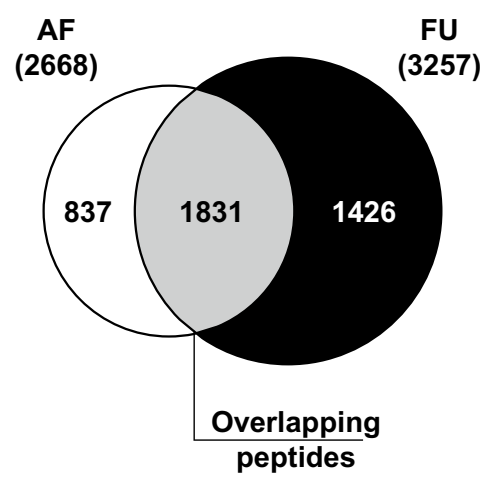

C

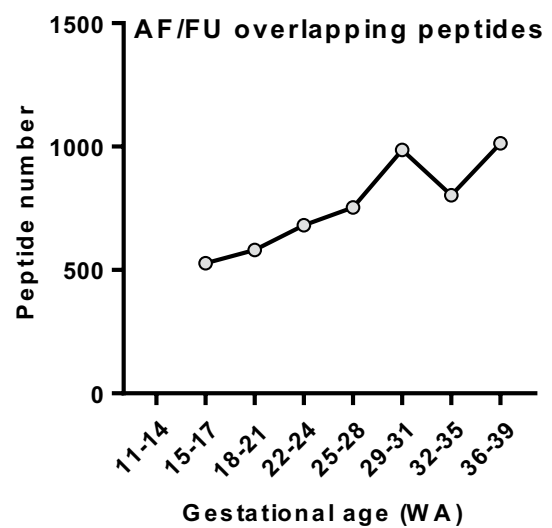

B

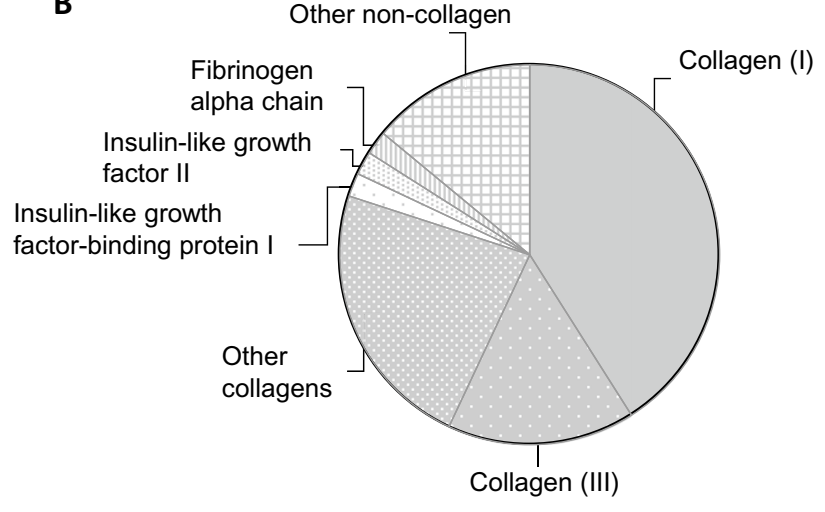

D

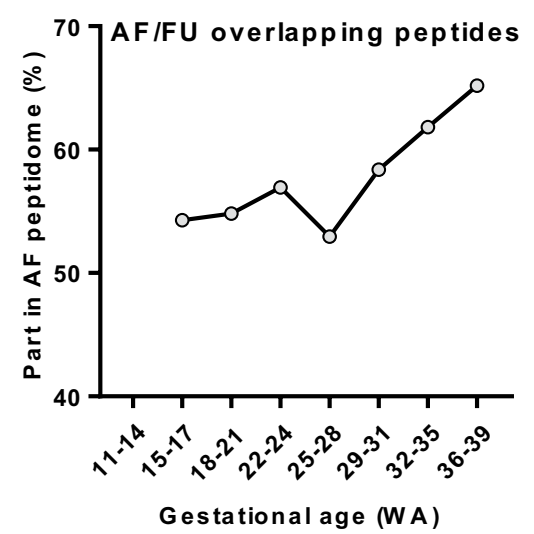

Figure 2. Overlap in the amniotic fluid and fetal urine peptidome. (A) Overlapping peptides in both amniotic fluid $(\mathrm{AF})$ and fetal urine $(\mathrm{FU})$, irrespective of gestational age. The total number of peptides identified is indicated in parentheses. (B) Protein origin of common peptides. (C) Gestational age evolution in the number of overlapping peptides (left) and percentage represented in the AF peptide content (right). (D) Gestational age evolution in the abundance in AF of overlapping peptides (left) and its percentage represented in the AF peptide abundance (right).

To the best of our knowledge, the present report is the first that compared amniotic and fetal urinary peptidome. Therefore, data showing that $>50 \%$ peptides present in $\mathrm{AF}$ were also identified in $\mathrm{FU}$ expand the current knowledge in the field.

It has already been shown that both fetal diuresis and (adult) urinary proteins predominantly participated to $\mathrm{AF}$ volume ${ }^{16,19}$ and proteome ${ }^{17}$, respectively. Here, we added the urinary peptidome component as an important contributor in $\mathrm{AF}$ peptide content, thereby supporting the concept that $\mathrm{AF}$ production is strongly driven by $\mathrm{FU}$ excretion.

We considered that peptides detected both in FU and AF originated from FU. Nevertheless, we cannot rule out the possibility that some of these peptides derived from an additional source. AF peptides potentially also result from fetal respiratory tract secretions, recognized as another significant source of AF. Although no information on the peptide content of fetal lung fluid is available, such hypothesis has to be taken account since collagen (I) (from which most $\mathrm{AF} / \mathrm{FU}$ sequenced common peptides are derived) is an abundant matrix component in the developing lung ${ }^{20,21}$. In contrast, for the 67 peptides displaying a significant positive correlation of abundance in $\mathrm{AF}$ and $\mathrm{FU}$, any origin other than urine can reasonably be excluded.

Almost half of FU peptides (1426 peptides) appear to be specific for FU since undetectable in AF (Fig. 2A). These peptides were mainly (in number and abundance) derived from the different collagen chains. They were also less abundant in urine than the AF/FU overlapping peptides (Fig. S2A). Their absence in AF may seem surprising at first glance since the urinary tract opens into the amniotic cavity. However, they might be present in AF but diluted below the detection limits (Fig. S2A). Moreover, some FU specific peptides can be digested by peptidases present downstream the bladder from where FU samples were collected. To evaluate this hypothesis, we performed a simulation using the ExPaSy software (https://web.expasy.org/peptide_cutter/) $)^{22}$. Results showed that 19 out of 22 fragments of Collagen alpha-1(I) chain exclusively found in FU could be degraded by enzymes potentially present in AF such pepsin, trypsin, chymotrypsin and Arg-C proteinase ${ }^{23,24}$ (Table S4).

A total of 837 peptides was exclusively present in AF. These included peptides of collagens (69 peptides), apolipoprotein (5 peptides), complement (5 peptides) and inter-alpha-trypsin inhibitor heavy chain $\mathrm{H} 4$ (5 peptides). 
A
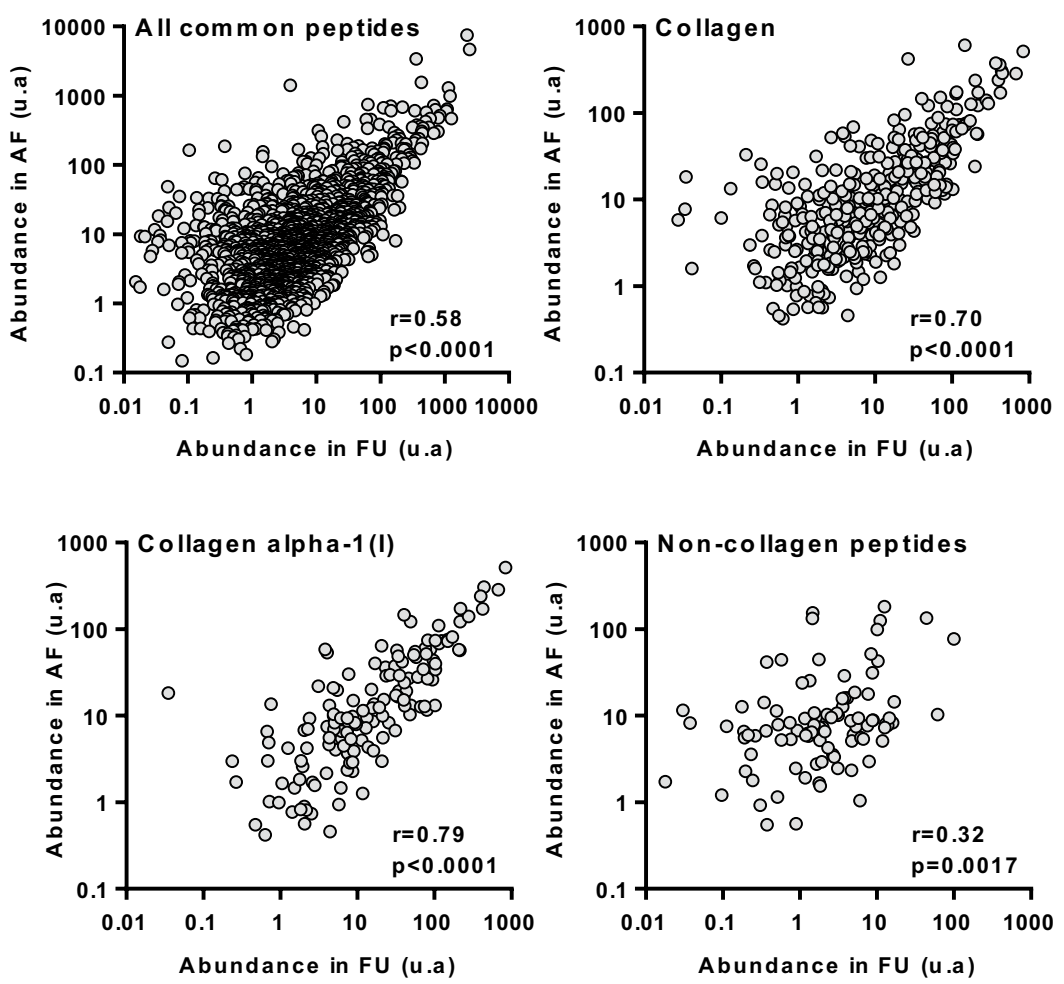

B

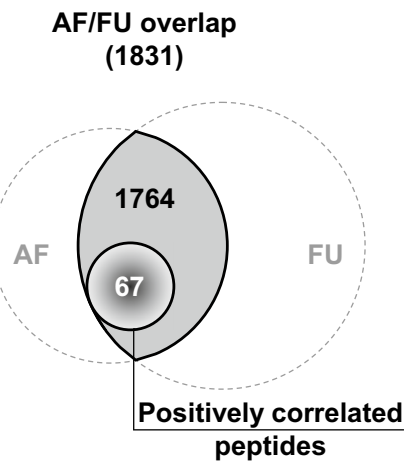

C
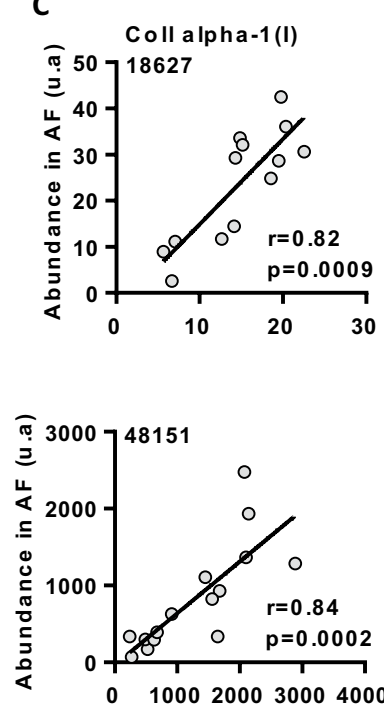

Abundance in FU (u.a)
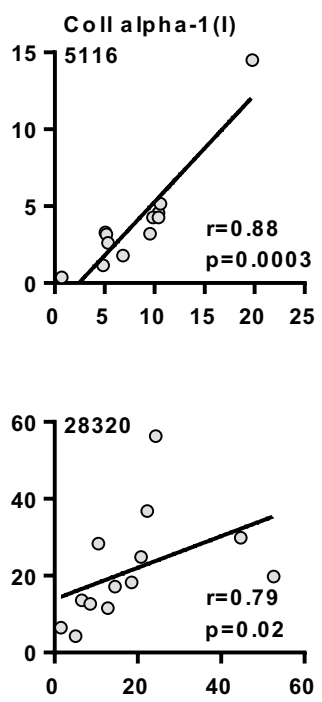

Abundance in FU (u.a)

Figure 3. Analysis of matched amniotic fluid and fetal urine samples. (A) Correlation of the average abundance in AF of all, collagen, collagen alpha-1(I) and non-collagen overlapping peptides with their average abundance in FU irrespective of gestational age. r, Spearman correlation coefficient. (B) Number of common peptides whose abundance is correlated in matched amniotic fluid (AF) and fetal urine (FU) from a subset of 16 fetuses. The total number of peptides in the overlap is indicated in parentheses. (C) Correlation between abundance of 4 representative overlapping peptides. $r$, Spearman correlation coefficient. 18627, fragment of collagen alpha-1(I) chain; 5116, fragment of collagen alpha-1(I) chain; 48151 and 28320, non-sequenced peptides. Coll, collagen.

One fragment of heparin cofactor 2, 4 fragments of thymosin beta 4, and 1 cornulin sequence were among the most abundant specific AF peptides. It is unlikely that these peptides would have escaped detection in the urine due to low abundance since their abundance in AF was similar to that of AF/FU common peptides (Fig. S2B). 
A

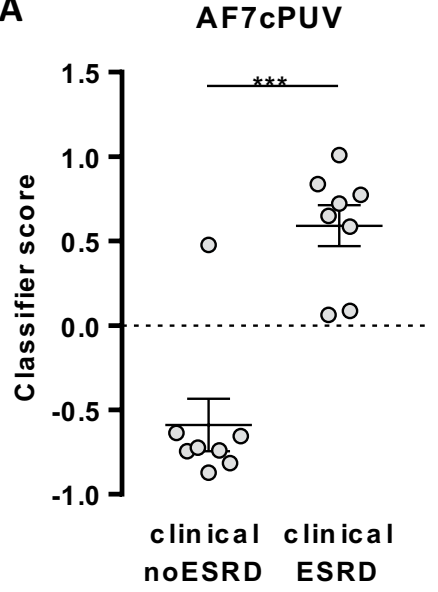

\section{B}

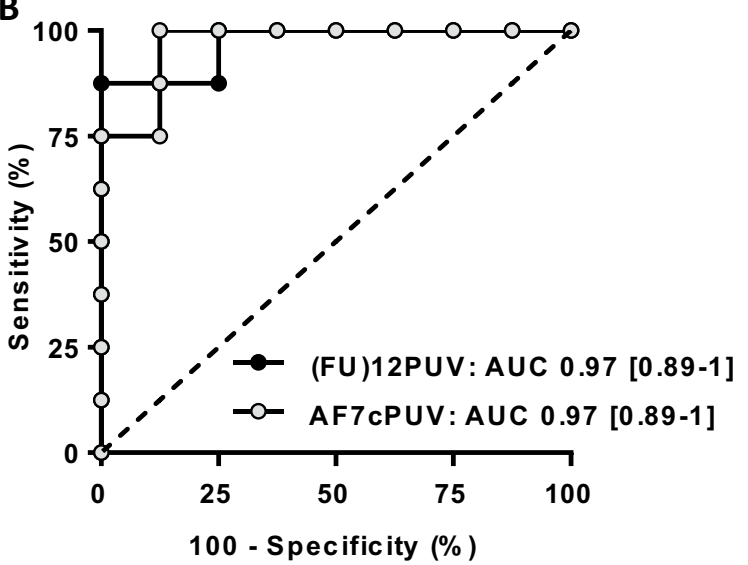

(FU)12PUV

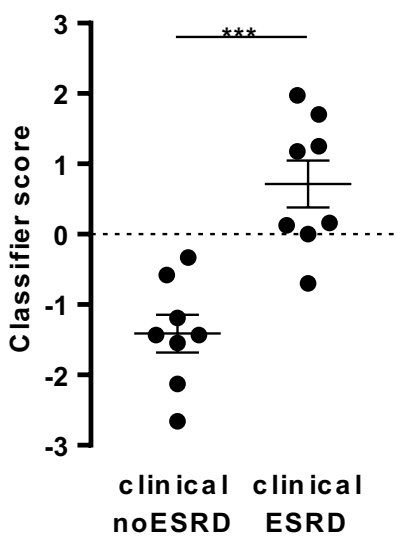

C

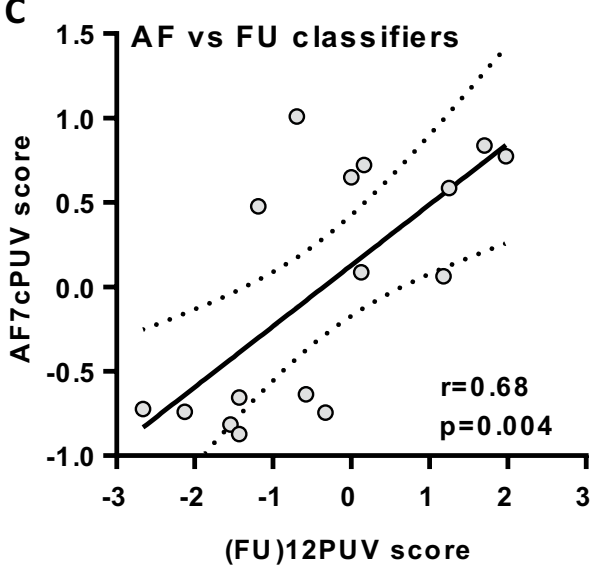

Figure 4. Potential of amniotic peptides for the prediction of renal survival in PUV fetuses. (A) Scatter dot plots showing AF7cPUV and (FU)12PUV classifier-based scores in 16 PUV patients from the validation cohort. The dotted horizontal line indicates the cutoff score ( 0 for both AF7cPUV and (FU)12PUV). A score $>$ cutoff suggested early development of ESRD whereas a score $<$ cutoff predicted fetus in the noESRD group. Data are means \pm SEM. ${ }^{* * *} \mathrm{p}<0.001$ versus noESRD according to Mann-Whitney test. (B) Compared ROC curves of AF7cPUV and (FU)12PUV. Brackets indicated confidence intervals for AUC. (C) Correlation between AF7cPUV and (FU)12PUV based scores.

However, a number of these peptides could derive from other fetal sources or from maternal circulation instead of FU. They could also result from the digestion of urinary proteins, as demonstrated by an analysis of the cleavage site specificity performed using Proteasix algorithm (http://proteasix.cs.man.ac.uk/25 that yielded a list of 27 potential AF proteases possibly liable for the generation of $129 \mathrm{AF}$ restricted sequenced peptides (Table S5).

A recent study has proposed that the majority of urinary peptides may originate from the kidney ${ }^{26}$. By extension, our results suggest that the AF peptidome may represent to a certain extent processes occurring in the developing kidney. This hypothesis is supported by our proof-of-concept study that shows that focusing on the $\mathrm{AF}$ abundance of $67 \mathrm{AF} / \mathrm{FU}$ correlated common peptides allowed prediction of postnatal renal survival in fetuses with posterior urethral valves. We recently started the ANTENATAL study (NCT0311621727), an international, prospective and case-control study aiming to recruit more than 400 fetuses with PUV over a range of 4 years for the multicenter validation of the initially identified FU peptide-based classifier ${ }^{10}$. The ANTENATAL study will be an excellent opportunity to reevaluate in a much larger dataset the potential of focusing on urine-derived peptides in $\mathrm{AF}$ for diagnosis of fetal renal disease.

Other studies have explored the AF content and origin. However, while most studies were based on a limited set of fetuses ${ }^{28-31}$, we have here collected an important number of both AF and FU samples (216 and 64, respectively). This high sample number ranging throughout second and third trimester of pregnancy also allowed a precise vision of gestational age-dependent changes in AF peptide content. Moreover, while a previous study investigated the urinary origin of $\mathrm{AF}$ proteins comparing $\mathrm{AF}$ to adult urine ${ }^{17}$, in this study we compared for the first time AF to FU, allowing a much more physiological comparison of the urine and AF content. Finally, a subset of matched $\mathrm{AF}$ and $\mathrm{FU}$ samples was available in which the peptidome was analyzed under the same operating conditions, within the same laboratory, with the same CE-MS device, by a unique operator and over a short interval of time, thereby allowing a robust comparison. 
One limitation of this study is that both AF and FU samples were mainly collected from pathological (mainly CAKUT) pregnancies, potentially hampering the generalization of the conclusions. Moreover, we were not able to explore the AF peptidome at the beginning of the pregnancy as amniocentesis is rarely done before the end of the first trimester. However, it is expected that the AF composition is similar to that of fetal plasma during this period which contains potentially less biomarkers of fetal kidney development. Finally, only $23 \%$ and $19 \%$ of the AF and FU peptides were sequenced, respectively. Additional sequence analysis could shed further light on the overlap.

In conclusion, these results suggest that AF peptidome can be considered as a good surrogate of the FU peptidome and thus represent a potential source of biomarkers for developmental kidney disease.

\section{Methods}

Patients. The present study is a retrospective analysis of data previously reported by our laboratory ${ }^{10,13,14}$. It included 13 healthy fetuses ${ }^{14}, 47$ fetuses with congenital CMV infection ${ }^{13}$ and 220 fetuses with CAKUT ${ }^{10,14}$ recruited in multidisciplinary prenatal diagnosis centers in France and Belgium. Based on the distribution of available samples the gestational period was divided in eight periods: 11-14 WA, 15-17 WA, 18-21 WA, 22-24 WA, 25-28 WA, 29-31 WA, 32-35 WA and 36-39 WA (Fig. S1).

In France, research based on retrospective data is excluded from the law relating to research involving the human subjects (known as the Jardé law) because it does not involve individuals but data. In this case, ethical opinion is not required. The present study related exclusively to the re-use of data already collected and published (available as supplementary data ${ }^{10,13,14}$ ) and required in no way access to the identifiable patient data since publicly available in the publications. Therefore, it does not fall into the category of research covered by the Jardé law as mentioned above. For this reason, we did not seek ethics approval.

Peptidomics data. Details for sample collection and preparation, CE-MS-based peptidome analysis and associated data processing, as well as peptide sequencing, have been previously described in the original publications $s^{10,13,14}$ and can be found in the Supplementary methods. In the present study, only peptides that were detected in one biological fluid (AF or FU) with a minimum frequency of $50 \%$ in at least one gestational period were investigated.

AF peptide based classifier. The potential of AF peptides to prognosticate the severity of developmental renal disease was evaluated in a subset of 30 fetuses with PUV. The judgment criterion was the development-or not-of ESRD at 2 years postnatally ${ }^{10,18}$. The ESRD group included PUV patients (14/30) that died in the neonatal period due to ESRD or that were subjected to TOP due to the severity of renal lesions. The noESRD group was composed of PUV patients (16/30) that led to liveborn infants having glomerular filtration rate (estimated using Schwartz formula) $>15 \mathrm{ml} / \mathrm{min}$ at 2 years of life. Fetuses were divided into independent discovery and validation subsets for generation and subsequent testing of the omics-based prognosis classifier, respectively. Significant $\mathrm{AF}$ peptides of $\mathrm{FU}$ origin were modelled using an in-house developed tool ${ }^{32}$ into a Random Forest (RF)-based classifier (RF package of $\mathrm{R}^{33}$ ) to generate the prognostic AF7cPUV classifier. The classifier was optimized for the prognosis of the 14 patients of the training set. The cutoff was determined as the score which allowed the optimal discrimination of PUV patients with early ERSD from PUV patients without postnatal ESRD. The number of trees was fixed to 1000 and the other parameters were kept as default values. Predictive performance was assessed by calculating sensitivity, specificity and AUC using MedCalc (version 16.8, MedCalc Software). Twosided confidence $95 \%$ CI were calculated to quantify the statistical precision of the measurements.

Statistical analyses. Comparison of peptide abundances, molecular masses and classifier-based scores of PUV fetuses were performed using a Mann-Whitney test. Effect of gestational age on peptide abundance was assessed according to One-way Anova. Relation between peptide abundances in AF and FU was evaluated performing Spearman correlation test. Peptides with significantly different AF abundance in PUV patients with early ESRD compared to PUV fetuses without ESRD at 2 years postnatally were selected by Wilcoxon analysis. To assess the discriminatory ability of the classifier, the hypothesis that the AUC is 0.5 (value indicating "no discrimination") was tested ${ }^{34}$. In all cases, two-sided tests were conducted and $\mathrm{p}<0.05$ was considered as statistically significant.

Received: 10 July 2020; Accepted: 27 November 2020

Published online: 10 December 2020

\section{References}

1. Good, D. M. et al. Naturally occurring human urinary peptides for use in diagnosis of chronic kidney disease. Mol. Cell. Proteom. MCP 9, 2424-2437. https://doi.org/10.1074/mcp.M110.001917 (2010).

2. Klein, J., Bascands, J. L., Mischak, H. \& Schanstra, J. P. The role of urinary peptidomics in kidney disease research. Kidney Int. 89, 539-545. https://doi.org/10.1016/j.kint.2015.10.010 (2016).

3. Metzger, J. et al. Urinary excretion of twenty peptides forms an early and accurate diagnostic pattern of acute kidney injury. Kidney Int. 78, 1252-1262. https://doi.org/10.1038/ki.2010.322 (2010).

4. Schanstra, J. P. \& Mischak, H. Proteomic urinary biomarker approach in renal disease: from discovery to implementation. Pediatr. Nephrol. 30, 713-725. https://doi.org/10.1007/s00467-014-2790-y (2015).

5. Brown, C. E. et al. Urinary proteomic biomarkers to predict cardiovascular events. Proteom. Clin. Appl. 9, 610-617. https://doi. org/10.1002/prca.201400195 (2015). 
6. Neisius, U. et al. Urine proteomics in the diagnosis of stable angina. BMC Cardiovasc. Disord. 16, 70. https://doi.org/10.1186/s1287 2-016-0246-y (2016).

7. von zur Muhlen, C. et al. Urine proteome analysis reflects atherosclerotic disease in an ApoE-/- mouse model and allows the discovery of new candidate biomarkers in mouse and human atherosclerosis. Mol. Cell. Proteom. MCP 11, M111013847. https:// doi.org/10.1074/mcp.M111.013847 (2012).

8. Frantzi, M. et al. Discovery and validation of urinary biomarkers for detection of renal cell carcinoma. J. Proteom. 98, 44-58. https ://doi.org/10.1016/j.jprot.2013.12.010 (2014).

9. Frantzi, M. et al. Development and validation of urine-based peptide biomarker panels for detecting bladder cancer in a multicenter study. Clin. Cancer Res. Off. J. Am. Assoc. Cancer Res. 22, 4077-4086. https://doi.org/10.1158/1078-0432.CCR-15-2715 (2016).

10. Klein, J. et al. Fetal urinary peptides to predict postnatal outcome of renal disease in fetuses with posterior urethral valves (PUV). Sci. Transl. Med. 5, 198ra106. https://doi.org/10.1126/scitranslmed.3005807 (2013).

11. Buffin-Meyer, B. et al. A single-center study to evaluate the efficacy of a fetal urine peptide signature predicting postnatal renal outcome in fetuses with posterior urethral valves. Pediatr. Nephrol. 35, 469-475. https://doi.org/10.1007/s00467-019-04390-9 (2020).

12. Klein, J. et al. Clinical proteomics in obstetrics and neonatology. Expert Rev. Proteom. 11, 75-89. https://doi.org/10.1586/14789 450.2014.872564 (2014).

13. Desveaux, C. et al. Identification of symptomatic fetuses infected with cytomegalovirus using amniotic fluid peptide biomarkers. PLoS Pathog. 12, e1005395. https://doi.org/10.1371/journal.ppat.1005395 (2016).

14. Klein, J. et al. Amniotic fluid peptides predict postnatal renal survival in developmental renal disease. Kidney international (in press).

15. Dubil, E. A. \& Magann, E. F. Amniotic fluid as a vital sign for fetal wellbeing. Aust. J. Ultrasound Med. 16, 62-70. https://doi. org/10.1002/j.2205-0140.2013.tb00167.x (2013).

16. Underwood, M. A., Gilbert, W. M. \& Sherman, M. P. Amniotic fluid: not just fetal urine anymore. J. Perinatol. Off. J. Calif. Perinatal Assoc. 25, 341-348. https://doi.org/10.1038/sj.jp.7211290 (2005).

17. Zhao, M. et al. A comparative proteomics analysis of five body fluids: plasma, urine, cerebrospinal fluid, amniotic fluid, and saliva. Proteom. Clin. Appl. 12, e1800008. https://doi.org/10.1002/prca.201800008 (2018).

18. Buffin-Meyer, B. et al. Combination of the fetal urinary metabolome and peptidome for the prediction of postnatal renal outcome in fetuses with PUV. J. Proteom. 184, 1-9. https://doi.org/10.1016/j.jprot.2018.06.012 (2018).

19. Brace, R. A. \& Cheung, C. Y. Pre-delivery changes in amniotic fluid volume and composition in sheep. J. Soc. Gynecol. Investig. 12, 396-401. https://doi.org/10.1016/j.jsgi.2005.04.004 (2005).

20. Davila, R. M., deMello, D. \& Crouch, E. C. Ontogeny of type I procollagen expression during human fetal lung development. Am. J. Physiol. 268, L309-320. https://doi.org/10.1152/ajplung.1995.268.2.L309 (1995).

21. Zhou, Y. et al. Extracellular matrix in lung development, homeostasis and disease. Matrix Biol. J. Int. Soc. Matrix Biol. 73, 77-104. https://doi.org/10.1016/j.matbio.2018.03.005 (2018).

22. Gasteiger, E. et al. ExPASy: The proteomics server for in-depth protein knowledge and analysis. Nucleic Acids Res. 31, 3784-3788. https://doi.org/10.1093/nar/gkg563 (2003).

23. Carrere, J., Muller, F., Boue, A., Guy-Crotte, O. \& Figarella, C. Levels and molecular forms of immunoreactive trypsin and chymotrypsin in amniotic fluids from normal and cystic fibrosis fetus: evidence for a lack of activation of proteolytic zymogens in cystic fibrosis fetus. J. Pediatr. Gastroenterol. Nutr. 14, 198-203. https://doi.org/10.1097/00005176-199202000-00014 (1992).

24. Pocknee, R. C. \& Abramovich, D. R. Origin and levels of trypsin in amniotic fluid throughout pregnancy. Br. J. Obstet. Gynaecol. 89, 142-144. https://doi.org/10.1111/j.1471-0528.1982.tb04681.x (1982).

25. Klein, J. et al. Proteasix: a tool for automated and large-scale prediction of proteases involved in naturally occurring peptide generation. Proteomics 13, 1077-1082. https://doi.org/10.1002/pmic.201200493 (2013).

26. Magalhaes, P. et al. Comparison of urine and plasma peptidome indicates selectivity in renal peptide handling. Proteom. Clin. Appl. 12, e1700163. https://doi.org/10.1002/prca.201700163 (2018).

27. Buffin-Meyer, B. et al. The ANTENATAL multicenter study to predict postnatal renal outcome in fetuses with posterior urethral valves: objectives and design. Clin. Kidney J. (in press).

28. Cho, C. K., Shan, S. J., Winsor, E. J. \& Diamandis, E. P. Proteomics analysis of human amniotic fluid. Mol. Cell. Proteom. MCP 6, 1406-1415. https://doi.org/10.1074/mcp.M700090-MCP200 (2007).

29. Cho, C. K., Smith, C. R. \& Diamandis, E. P. Amniotic fluid proteome analysis from Down syndrome pregnancies for biomarker discovery. J. Proteome Res. 9, 3574-3582. https://doi.org/10.1021/pr100088k (2010).

30. Tsangaris, G. T. et al. The normal human amniotic fluid supernatant proteome. Vivo 20, 479-490 (2006).

31. Park, S. J. et al. Proteome analysis of human amnion and amniotic fluid by two-dimensional electrophoresis and matrix-assisted laser desorption/ionization time-of-flight mass spectrometry. Proteomics 6, 349-363. https://doi.org/10.1002/pmic.200500084 (2006).

32. Boizard, F. et al. A capillary electrophoresis coupled to mass spectrometry pipeline for long term comparable assessment of the urinary metabolome. Sci. Rep. 6, 34453. https://doi.org/10.1038/srep34453 (2016).

33. Liaw, A. \& Wiener, M. Classification and regression by random forest. R News 2, 18-22 (2002).

34. Hanley, J. A. \& McNeil, B. J. The meaning and use of the area under a receiver operating characteristic (ROC) curve. Radiology 143, 29-36. https://doi.org/10.1148/radiology.143.1.7063747 (1982).

\section{Acknowledgements}

Part of the work was financed by grants from the "Fondation pour la Recherche Médicale" (Grant number DEQ20170336759), from the "Agence de la Biomédecine" (METAPhOR project) and from the "Filière de santé des maladies rénales rares, ORKiD".

\section{Author contributions}

J.P.S. and B.B.M. conceptualized the study. C.F., B.B., I.G., P.M., P.Z., J.K., J.P.S. and B.B.M. contributed to the acquisition, analysis and interpretation of the data. S.D., F.M. and S.D. contributed with patients and follow-up data. J.P.S. and B.B.M. wrote the first original draft. C.F., P.M., S.D., P.Z. and J.K. revised the manuscript critically for important intellectual content. All authors read and approved the final version of the manuscript.

\section{Competing interests}

I. Golovko, P. Magalhães and P. Zürbig and are employed by Mosaiques Diagnostics GmbH. The other authors declare no competing interests. 


\section{Additional information}

Supplementary information (SI) The online version contains supplementary material availlable at https://doi. org/10.1038/s41598-020-78730-3.

Correspondence and requests for materials should be addressed to J.P.S. or B.B.-M.

Reprints and permissions information is available at www.nature.com/reprints.

Publisher's note Springer Nature remains neutral with regard to jurisdictional claims in published maps and institutional affiliations.

(c) (1) Open Access This article is licensed under a Creative Commons Attribution 4.0 International cc) License, which permits use, sharing, adaptation, distribution and reproduction in any medium or format, as long as you give appropriate credit to the original author(s) and the source, provide a link to the Creative Commons licence, and indicate if changes were made. The images or other third party material in this article are included in the article's Creative Commons licence, unless indicated otherwise in a credit line to the material. If material is not included in the article's Creative Commons licence and your intended use is not permitted by statutory regulation or exceeds the permitted use, you will need to obtain permission directly from the copyright holder. To view a copy of this licence, visit http://creativecommons.org/licenses/by/4.0/.

(C) The Author(s) 2020 